\title{
Coupling-constant expression and exact relations for the kinetic-energy functional in pair-density-functional theory
}

\author{
Katsuhiko Higuchi \\ Graduate School of Advanced Sciences of Matter, Hiroshima University, Higashi-Hiroshima 739-8527, Japan \\ Masahiko Higuchi \\ Department of Physics, Faculty of Science, Shinshu University, Matsumoto 390-8621, Japan \\ (Received 28 December 2011; published 11 June 2012)
}

\begin{abstract}
We derive the coupling-constant expression for the kinetic energy (KE) functional of the pair-density-functional theory. Such the expression can be effectively used in developing the approximate KE functional. Indeed, on the basis of this expression, we present an approximate form of the KE functional by means of the perturbation theory. Furthermore, as the first step of an alternative way of obtaining the approximate KE functional, we derive several relations and bounds for the KE functional by investigating how the KE functional changes with the nonuniform scaling, rotation, and translation of electron coordinates. Such relations and bounds can be utilized as restrictive conditions on the approximate form. Thus, the coupling-constant expression, relations and bounds obtained here provide useful tools for devising various types of the approximate KE functional that is indispensable for advancing the pair-density-functional theory.
\end{abstract}

DOI: 10.1103/PhysRevA.85.062508

PACS number(s): 31.15.ve, 31.15.xt, 71.15.Mb

\section{INTRODUCTION}

The pair density (PD) corresponds to the diagonal element of the second-order reduced density matrix and has more information about the electron correlation than the electron density [1-6]. Therefore, the PD-functional theory [7-44], which can reproduce the ground-state $\mathrm{PD}$, has recently attracted particular interest as a potential scheme that goes beyond the conventional density-functional theory (DFT) $[45,46]$. The PD-functional theory was first proposed by Ziesche $[7,8]$. Since then, two kinds of problems make it difficult for us to perform actual calculations of the PD-functional theory. One is related to the $N$-representability problem of the PD [1-6,26-39]. In carrying out a variational search for the ground-state PD, the search region of PDs should be the set of the $N$-representable PDs. However, the necessary and sufficient conditions for the $N$-representability of the PD have not yet been known in a practical form. We have recently attempted to utilize as the search region of PDs the set of PDs that come from some kind of antisymmetric wave functions [20-24]. More recently, we have proposed an efficient method to extend the search region within the set of $N$-representable PDs [25]. Specifically, the search region of PDs is extended by adding the uniformly scaled PDs to elements of the search region. The validity has been confirmed by numerical calculations [25]. Thus, the method of how to set up the search region of PDs, which is related to the computational time [47], is one of two problems in the PD-functional theory.

The other problem is related to the kinetic energy (KE) functional of the PD-functional theory. Since the KE cannot be expressed by the PD alone, some approximation is needed for the KE functional [39-44]. For the purpose of solving this issue, it is helpful to review how to develop the approximate form of the exchange-correlation (xc) energy functional of the DFT and its extensions [48-52]. Generally, there are two strategies to develop the approximate form of the xc energy functional [52]. One is to utilize the coupling-constant expression for the xc energy functional. For example, the local density approximation [46], average-density approximation [53-56], and weighted-density approximation [54-58] are classified into approximations that are developed along with this strategy. The other is to utilize relations and bounds, which are fulfilled by the exact xc energy functional, as the restrictive conditions. The generalized gradient approximation [59-63], density-moment approximation [64-69], and vorticity expansion approximation [70-73] are developed along with this strategy. It is expected that these two strategies are also applicable to developing the approximate form of the $\mathrm{KE}$ functional of the PD-functional theory.

In this paper, we take the abovementioned two strategies for the development of the approximate form of the KE functional. Concerning the first strategy, we derive the coupling-constant expression for the KE functional. On the basis of this expression, an approximate form of the $\mathrm{KE}$ functional is also presented using the perturbation theory.

As for the second strategy, two kinds of relations and bounds for the KE functional of the PD theory have been derived so far [11,24]. Several approximate KE functionals have been developed by requiring them to fulfill two relations and bounds $[24,25]$. The validity of the approximate forms is checked by actual calculations, and it is shown that there remains room for improvement [24,25]. Since relations and bounds are regarded as the necessary conditions on the approximate functional, more and/or stronger necessary conditions are desired for the improvement of the approximate functional. In this paper, we derive several kinds of relations and bounds by investigating the behavior of the KE functional under the nonuniform scaling, rotation, and translation of electron coordinates. The usefulness of these relations and bounds is also discussed.

Organization of this paper is as follows. In Sec. II, along with the first strategy, the KE functional is given in the coupling-constant expression. The approximate form based on the expression is also discussed. In Sec. III, several kinds of relations and bounds are given along with the second strategy. Also, examples of using relations and bounds are presented. Finally, some concluding remarks are given in Sec. IV. 


\section{COUPLING-CONSTANT EXPRESSION FOR THE KE FUNCTIONAL}

In this section, we derive the exact expression for the KE functional by means of the coupling-constant integration. Let us consider the system where the Hamiltonian is given by

$$
\hat{H}=\hat{T}+\hat{W}+\hat{V},
$$

where $\hat{T}, \hat{W}$, and $\hat{V}$ are operators of the kinetic, electronelectron interaction, and external potential energies, respectively. Due to the Hohenberg-Kohn theorem with respect to the PD [7,8], the ground-state wave function can be denoted as $\left|\Psi\left[\gamma^{(2)}\right]\right\rangle$, where $\gamma^{(2)}$ is the ground-state PD of the system. The KE functional of the PD-functional theory is given by

$$
T\left[\gamma^{(2)}\right]=\left\langle\Psi\left[\gamma^{(2)}\right]|\hat{T}| \Psi\left[\gamma^{(2)}\right]\right\rangle_{\Omega},
$$

where $\left|\Psi\left[\gamma^{(2)}\right]\right\rangle$ is normalized to unity in the volume $\Omega$ and the subscript of the angular bracket in Eq. (2) means that integrals are performed over the volume $\Omega$. In the previous scheme $[20,22,23]$, the noninteracting reference system is introduced so as to reproduce the variationally best PD within the set of PDs that are constructed from the single Slater determinants (SDs). The Hamiltonian of the noninteracting reference system is given by

$$
\hat{H}_{\mathrm{eff}}=\hat{T}+\hat{V}_{\mathrm{eff}}^{\mathrm{int}}+\hat{V}
$$

where $\hat{V}_{\mathrm{eff}}^{\mathrm{int}}$ denotes the effective potential of the electronelectron interaction, which is similar to that of the correlated Hartree-Fock equation [20,22,23]. The explicit form of $\hat{V}_{\text {eff }}^{\text {int }}$ is given so that the correct electron density of the ground state is reproduced in the noninteracting reference system [22,23]. This fact will be used later.

In order to derive the coupling-constant expression for $T\left[\gamma^{(2)}\right]$, we consider the system, the Hamiltonian of which is given by

$$
\hat{H}_{\lambda}=\hat{T}+\lambda \hat{W}+(1-\lambda) \hat{V}_{\mathrm{eff}}^{\mathrm{int}}+\hat{V},
$$

where $\lambda$ takes a real value from 0 to 1 . This Hamiltonian coincides with Eqs. (1) and (3) in the cases of $\lambda=1$ and $\lambda=0$, respectively. If the ground-state energy and wave function for this Hamiltonian are denoted by $E_{\lambda}$ and $\left|\Psi_{\lambda}\right\rangle$, respectively, then we have

$$
E_{\lambda}=\left\langle\Psi_{\lambda}\left|\hat{H}_{\lambda}\right| \Psi_{\lambda}\right\rangle_{\Omega} .
$$

With the aid of the Hellmann-Feynman theorem, the derivative of $E_{\lambda}$ with respect to $\lambda$ leads to

$$
\frac{d E_{\lambda}}{d \lambda}=\left\langle\Psi_{\lambda}\left|\hat{W}-\hat{V}_{\mathrm{eff}}^{\mathrm{int}}\right| \Psi_{\lambda}\right\rangle_{\Omega}
$$

Integrating both sides of Eq. (6) with respect to $\lambda$, we get

$$
E_{1}-E_{0}=\int_{0}^{1}\left\langle\Psi_{\lambda}\left|\hat{W}-\hat{V}_{\mathrm{eff}}^{\mathrm{int}}\right| \Psi_{\lambda}\right\rangle_{\Omega} d \lambda
$$

The left-hand side of Eq. (7) can be rewritten using the Hohenberg-Kohn theorem of the PD-functional theory. Since $\left|\Psi_{1}\right\rangle$ denotes the ground-state wave function, we have $\left|\Psi_{1}\right\rangle=$ $\left|\Psi\left[\gamma^{(2)}\right]\right\rangle$ from the Hohenberg-Kohn theorem. Therefore, $E_{1}$ is given by

$$
\begin{aligned}
E_{1} & =\left\langle\Psi\left[\gamma^{(2)}\right]|\hat{T}+\hat{W}+\hat{V}| \Psi\left[\gamma^{(2)}\right]\right\rangle_{\Omega} \\
& =T\left[\gamma^{(2)}\right]+W\left[\gamma^{(2)}\right]+V\left[\gamma^{(2)}\right],
\end{aligned}
$$

where $W\left[\gamma^{(2)}\right]$ and $V\left[\gamma^{(2)}\right]$ are the expectation values of $\hat{W}$ and $\hat{V}$ with respect to $\left|\Psi\left[\gamma^{(2)}\right]\right\rangle$, respectively. Similarly to Eq. (8), $E_{0}$ can be rewritten as the functional of $\gamma_{S D}^{(2)}$ that stands for the ground-state PD of the noninteracting reference system. Since $\left|\Psi_{0}\right\rangle$ can be denoted by $\left|\Phi\left[\gamma_{S D}^{(2)}\right]\right\rangle$ with the aid of the Hohenberg-Kohn theorem for the noninteracting reference system, we have

$$
\begin{aligned}
E_{0} & =\left\langle\Phi\left[\gamma_{S D}^{(2)}\right]\left|\hat{T}+\hat{V}_{\mathrm{eff}}^{\mathrm{int}}+\hat{V}\right| \Phi\left[\gamma_{S D}^{(2)}\right]\right\rangle_{\Omega} \\
& =T_{s}\left[\gamma_{S D}^{(2)}\right]+V_{\mathrm{eff}}^{\mathrm{int}}\left[\gamma_{S D}^{(2)}\right]+V\left[\gamma_{S D}^{(2)}\right],
\end{aligned}
$$

where $T_{S}\left[\gamma_{S D}^{(2)}\right], V_{\mathrm{eff}}^{\mathrm{int}}\left[\gamma_{S D}^{(2)}\right]$, and $V\left[\gamma_{S D}^{(2)}\right]$ are the expectation values of $\hat{T}, \hat{V}_{\mathrm{eff}}^{\text {int }}$, and $\hat{V}$ with respect to $\left|\Phi\left[\gamma_{S D}^{(2)}\right]\right\rangle$, respectively. It should be noted that $\gamma_{S D}^{(2)}$ is the functional of $\gamma^{(2)}$. This is because $\gamma_{S D}^{(2)}$ is given as the variationally best PD that is determined for the real system [20,22,23]. Since the external potential is determined by $\gamma^{(2)}$ due to the Hohenberg-Kohn theorem, we can say that $\gamma_{S D}^{(2)}$ is expressed as the functional of $\gamma^{(2)}$, that is, $\gamma_{S D}^{(2)}\left[\gamma^{(2)}\right]$. Using the notation $\gamma_{S D}^{(2)}\left[\gamma^{(2)}\right]$ and substituting Eqs. (8) and (9) into Eq. (7), we obtain

$$
\begin{aligned}
T\left[\gamma^{(2)}\right]= & T_{S}\left[\gamma_{S D}^{(2)}\left[\gamma^{(2)}\right]\right]+\int_{0}^{1}\left\langle\Psi_{\lambda}\left|\hat{W}-\hat{V}_{\mathrm{eff}}^{\mathrm{int}}\right| \Psi_{\lambda}\right\rangle_{\Omega} d \lambda \\
& -W\left[\gamma^{(2)}\right]+V_{\mathrm{eff}}^{\mathrm{int}}\left[\gamma_{S D}^{(2)}\left[\gamma^{(2)}\right]\right],
\end{aligned}
$$

where we use the fact that the correct electron density of the ground state is reproduced in the noninteracting reference system [22]. Equation (10) is the coupling-constant expression for the KE functional of the PD-functional theory. One can use this expression as a starting point of developing the approximate form of $T\left[\gamma^{(2)}\right]$. It should be noted that Eq. (10) consists of the noninteracting $\mathrm{KE}$ and its correction terms, which is similar to the $\mathrm{KE}$ functional of the conventional DFT [74].

In order to develop the approximate form of $T\left[\gamma^{(2)}\right]$, the second term of Eq. (10) should be devised. The wave function $\left|\Psi_{\lambda}\right\rangle$ in it can be approximated by reference to the densityfunctional perturbation theory $[75,76]$. Namely, we decompose the Hamiltonian $\hat{H}_{\lambda}$ into the nonperturbative Hamiltonian $\hat{H}_{\text {eff }}$ and perturbation $\lambda\left(\hat{W}-\hat{V}_{\text {eff }}^{\text {int }}\right)$. If we denote the eigenfunction of $\hat{H}_{\text {eff }}$ by $\left|\Phi_{k}\right\rangle$, then we have

$$
\hat{H}_{\text {eff }}\left|\Phi_{k}\right\rangle=\varepsilon_{k}\left|\Phi_{k}\right\rangle
$$

where $\varepsilon_{k}$ is the eigenvalue for $\left|\Phi_{k}\right\rangle$, and $\left|\Phi_{0}\right\rangle$ corresponds to the ground-state wave function of the noninteracting reference system, that is, $\left|\Phi_{0}\right\rangle=\left|\Phi\left[\gamma_{S D}^{(2)}\left[\gamma^{(2)}\right]\right]\right\rangle$. With the aid of the perturbation theory, $\left|\Psi_{\lambda}\right\rangle$ is given by

$$
\begin{aligned}
\left|\Psi_{\lambda}\right\rangle= & \left|\Phi\left[\gamma_{S D}^{(2)}\left[\gamma^{(2)}\right]\right]\right\rangle \\
& +\lambda \sum_{k \neq 0} \frac{\left\langle\Phi_{k}\left|\hat{W}-\hat{V}_{\mathrm{eff}}^{\mathrm{int}}\right| \Phi\left[\gamma_{S D}^{(2)}\left[\gamma^{(2)}\right]\right]\right\rangle_{\Omega}}{\varepsilon_{0}-\varepsilon_{k}}\left|\Phi_{k}\right\rangle+\cdots
\end{aligned}
$$


Using Eq. (12), Eq. (10) is rewritten as

$$
\begin{aligned}
T\left[\gamma^{(2)}\right]= & T_{S}\left[\gamma_{S D}^{(2)}\left[\gamma^{(2)}\right]\right]-W\left[\gamma^{(2)}\right] \\
& +\left\langle\Phi\left[\gamma_{S D}^{(2)}\left[\gamma^{(2)}\right]\right]|\hat{W}| \Phi\left[\gamma_{S D}^{(2)}\left[\gamma^{(2)}\right]\right]\right\rangle_{\Omega} \\
& +\sum_{k \neq 0} \frac{\left|\left\langle\Phi_{k}\left|\hat{W}-\hat{V}_{\mathrm{eff}}^{\mathrm{int}}\right| \Phi\left[\gamma_{S D}^{(2)}\left[\gamma^{(2)}\right]\right]\right\rangle_{\Omega}\right|^{2}}{\varepsilon_{0}-\varepsilon_{k}}+\cdots
\end{aligned}
$$

Equation (13) is an alternative form of the exact expression for $T\left[\gamma^{(2)}\right]$. An approximate form of $T\left[\gamma^{(2)}\right]$ can be proposed on the basis of Eq. (13). As the lowest approximation, by neglecting the second- and higher-order terms of $\hat{W}-\hat{V}_{\mathrm{eff}}^{\text {int }}$, Eq. (13) is approximated to

$$
\begin{aligned}
T\left[\gamma^{(2)}\right] \approx & T_{S}\left[\gamma_{S D}^{(2)}\left[\gamma^{(2)}\right]\right]-\left\{W\left[\gamma^{(2)}\right]\right. \\
& \left.-\left\langle\Phi\left[\gamma_{S D}^{(2)}\left[\gamma^{(2)}\right]\right]|\hat{W}| \Phi\left[\gamma_{S D}^{(2)}\left[\gamma^{(2)}\right]\right]\right\rangle_{\Omega}\right\} .
\end{aligned}
$$

The second term of the right-hand side, that is, $W\left[\gamma^{(2)}\right]-$ $\left\langle\Phi\left[\gamma_{S D}^{(2)}\left[\gamma^{(2)}\right]\right]|\hat{W}| \Phi\left[\gamma_{S D}^{(2)}\left[\gamma^{(2)}\right]\right]\right\rangle_{\Omega}$, just corresponds to the correlated $\mathrm{KE}$ of the $\mathrm{PD}$-functional theory. Its magnitude is expected to be comparable to that of the correlation energy due to its form. This seems to be quite reasonable because the similar approximate formula is found in the conventional DFT, in which the magnitude of the correlated KE is shown to be approximately equal to that of the correlation energy functional $[6,77]$.

On the basis of Eq. (14), we may propose the approximate form of $T\left[\gamma^{(2)}\right]$. For instance, the first term may be approximated by the model functional for the noninteracting KE such as the Thomas-Fermi approximation, Weizsäcker formulas, and so on [6]. The second term may be approximated by the density functional of the correlation energy such as the local density approximation, the generalized gradient approximation, and so on [6]. Thus, an approximate form of $T\left[\gamma^{(2)}\right]$ can be obtained on the basis of the exact expression for $T\left[\gamma^{(2)}\right]$.

\section{EXACT RELATIONS AND BOUNDS FOR THE KE FUNCTIONAL}

\section{A. Exact relations and bounds for the KE functional by means of the nonuniform coordinate scaling of electrons}

In the conventional DFT and its extensions, the nonuniform coordinate scaling of electrons has been utilized in investigating properties of the xc energy functional $[52,78]$. The relations and bounds derived from the nonuniform coordinate scaling of electrons have been effectively used in devising the approximate form of the xc energy functional [59-63,70-73]. In this subsection, by following the cases of the conventional DFT [78] and current-densityfunctional theory (CDFT) [52], we derive exact relations and bounds for the KE functional of the PD-functional theory by means of the nonuniform coordinate scaling of electrons.

In Sec. III A 1, we first investigate properties of $\left|\Psi\left[\gamma^{(2)}\right]\right\rangle$ for general cases by means of the nonuniform coordinate scaling of electrons and then derive the fundamental properties of the KE functional. Using these, the exact relations and bounds for the KE functional can be derived, which are shown in Secs. III A 2 and III A 3. For convenience, the notations that are used in Sec. III A are summarized in Appendix A.

\section{Fundamental properties of the KE functional}

Let us consider the transformation of electron coordinates $\mathbf{r}_{i}=\left(x_{i}, y_{i}, z_{i}\right)(i=1, \ldots, N)$ into $\left(\zeta_{x}^{-1} x_{i}, \zeta_{y}^{-1} y_{i}, \zeta_{z}^{-1} z_{i}\right)$, where $\zeta_{x}, \zeta_{y}$, and $\zeta_{z}$ are positive and real numbers, and $N$ denotes the number of electrons. This type of transformation is called a nonuniform coordinate scaling of electrons. With this nonuniform coordinate scaling, the wave function $\Psi\left(\mathbf{r}_{1}, \ldots, \mathbf{r}_{N}, \eta_{1}, \ldots, \eta_{N}\right)$ is transformed into

$\Psi\left(\zeta_{x} x_{1}, \zeta_{y} y_{1}, \zeta_{z} z_{1}, \ldots, \zeta_{x} x_{N}, \zeta_{y} y_{N}, \zeta_{z} z_{N}, \eta_{1}, \ldots, \eta_{N}\right)$

where $\eta_{i}$ denotes the spin coordinate of electrons. Normalizing Eq. (15) to unity, we define the scaled wave function as

$$
\Psi_{\zeta_{x} \zeta_{y} \zeta_{z}}^{x y z}\left(\mathbf{r}_{1}, \ldots, \mathbf{r}_{N}, \eta_{1}, \ldots, \eta_{N}\right)=\left(\zeta_{x} \zeta_{y} \zeta_{z}\right)^{N / 2} \Psi\left(\zeta_{x} x_{1}, \zeta_{y} y_{1}, \zeta_{z} z_{1}, \ldots, \zeta_{x} x_{N}, \zeta_{y} y_{N}, \zeta_{z} z_{N}, \eta_{1}, \ldots, \eta_{N}\right),
$$

where $\left(\zeta_{x} \zeta_{y} \zeta_{z}\right)^{N / 2}$ is the normalizing constant. Note that if $\Psi\left(\mathbf{r}_{1}, \ldots, \mathbf{r}_{N}, \eta_{1}, \ldots, \eta_{N}\right)$ is normalized to unity in the volume $\Omega$, then $\Psi_{\zeta_{x} \zeta_{y} \zeta_{z}}^{x}\left(\mathbf{r}_{1}, \ldots, \mathbf{r}_{N}, \eta_{1}, \ldots, \eta_{N}\right)$ is normalized to unity in the volume $\Omega^{\prime}=\Omega /\left(\zeta_{x} \zeta_{y} \zeta_{z}\right)$. If we denote the PDs that are

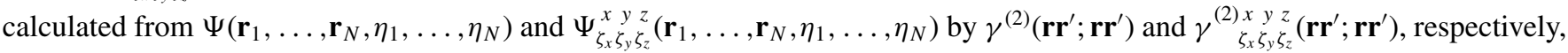

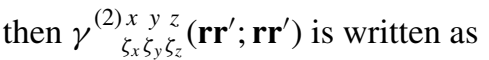

$$
\gamma_{\zeta_{x} \zeta_{y} \zeta_{z}}^{(2) x y}\left(\mathbf{r r}^{\prime} ; \mathbf{r r}^{\prime}\right)=\left(\zeta_{x} \zeta_{y} \zeta_{z}\right)^{2} \gamma^{(2)}\left(\zeta_{x} x, \zeta_{y} y, \zeta_{z} z, \zeta_{x} x^{\prime}, \zeta_{y} y^{\prime}, \zeta_{z} z^{\prime} ; \zeta_{x} x, \zeta_{y} y, \zeta_{z} z, \zeta_{x} x^{\prime}, \zeta_{y} y^{\prime}, \zeta_{z} z^{\prime}\right)
$$

$\gamma_{\zeta_{x} \zeta_{y} \zeta_{z}}^{(2) x} \quad\left(\mathbf{r r}^{\prime} ; \mathbf{r r}^{\prime}\right)$ is called a scaled PD, the domain of which is within the volume $\Omega^{\prime}$. In order to derive properties for $T\left[\gamma^{(2)}\right]$, we introduce the following functional:

$$
T_{\alpha \beta \gamma}\left[\gamma^{(2)}\right]=\operatorname{Min}_{\Psi \rightarrow \gamma^{(2)}}\left\langle\Psi\left|\alpha \hat{T}_{x}+\beta \hat{T}_{y}+\gamma \hat{T}_{z}\right| \Psi\right\rangle_{\Omega}=\left\langle\Psi_{\alpha \beta \gamma}\left[\gamma^{(2)}\right]\left|\alpha \hat{T}_{x}+\beta \hat{T}_{y}+\gamma \hat{T}_{z}\right| \Psi_{\alpha \beta \gamma}\left[\gamma^{(2)}\right]\right\rangle_{\Omega},
$$

where $\alpha, \beta$, and $\gamma$ are real numbers that enlarge or reduce the components of the $\mathrm{KE}$ anisotropically and where $\left|\Psi_{\alpha \beta \gamma}\left[\gamma^{(2)}\right]\right\rangle$ denotes the minimizing wave function. $\hat{T}_{a}(a=x, y, z)$ in Eq. (18) is given by

$$
\hat{T}_{a}=\sum_{i=1}^{N}\left(-\frac{\hbar^{2}}{2 m} \frac{\partial^{2}}{\partial a_{i}^{2}}\right) .
$$


It should be noted that $T_{\alpha \beta \gamma}\left[\gamma^{(2)}\right]$ coincides with $T\left[\gamma^{(2)}\right]$ in the case of $\alpha=\beta=\gamma=1$. In this case, we denote the minimizing

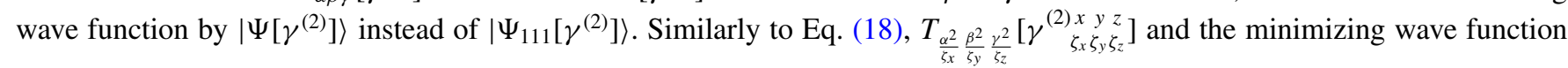

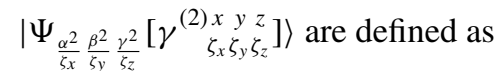

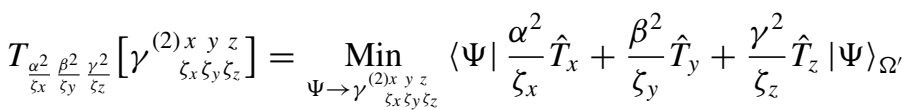

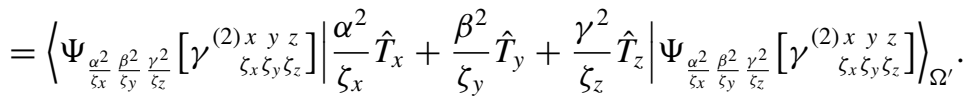

As shown in Appendix B, the following relation holds:

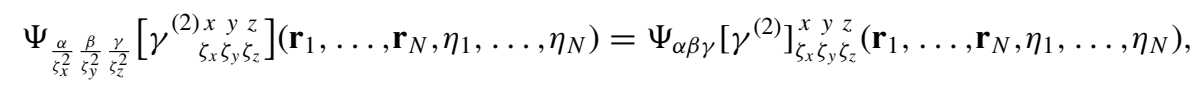

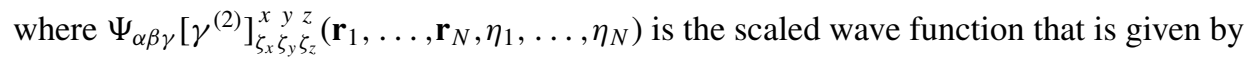

$$
\Psi_{\alpha \beta \gamma}\left[\gamma^{(2)}\right]_{\zeta_{x} \zeta_{y} \zeta_{z}}^{x} z_{z}\left(\mathbf{r}_{1}, \ldots, \mathbf{r}_{N}, \eta_{1}, \ldots, \eta_{N}\right)=\left(\zeta_{x} \zeta_{y} \zeta_{z}\right)^{N / 2} \Psi_{\alpha \beta \gamma}\left[\gamma^{(2)}\right]\left(\zeta_{x} x_{1}, \zeta_{y} y_{1}, \zeta_{z} z_{1}, \ldots, \zeta_{x} x_{N}, \zeta_{y} y_{N}, \zeta_{z} z_{N}, \eta_{1}, \ldots, \eta_{N}\right)
$$

Substituting $\alpha=\zeta_{x}^{2}, \beta=\zeta_{y}^{2}$, and $\gamma=\zeta_{z}^{2}$ into Eq. (21), we have

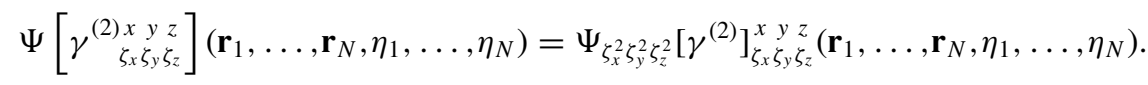

Using Eqs. (22) and (23), we obtain

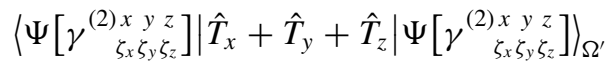

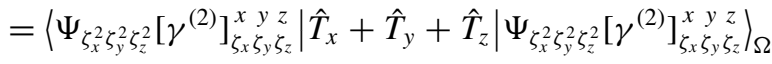

$$
\begin{aligned}
& =\left\langle\Psi_{\zeta_{x}^{2} \zeta_{y}^{2} \zeta_{z}^{2}}\left[\gamma^{(2)}\right]\left|\zeta_{x}^{2} \hat{T}_{x}+\zeta_{y}^{2} \hat{T}_{y}+\zeta_{z}^{2} \hat{T}_{z}\right| \Psi_{\zeta_{x}^{2} \zeta_{y}^{2} \zeta_{z}^{2}}\left[\gamma^{(2)}\right]\right\rangle_{\Omega} \text {. }
\end{aligned}
$$

This equation immediately leads to

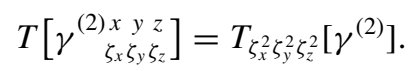

In the special case when $\zeta_{x}=\xi^{l}, \zeta_{y}=\xi^{m}$, and $\zeta_{z}=\xi^{n}$, where $\xi, l, m$, and $n$ are real numbers, Eq. (25) becomes

$$
T\left[\begin{array}{ccc}
(2) x & y & z \\
\xi^{l} \xi^{m} \xi^{n}
\end{array}\right]=T_{\xi^{2 l} \xi^{2 m} \xi^{2 n}\left[\gamma^{(2)}\right]}
$$

Furthermore, we consider the other type of properties of the KE functional. By differentiating both sides of Eq. (25) with respect to $\zeta_{a}(a=x, y, z)$ and by using the HellmannFeynman theorem, we have

$\frac{d}{d \zeta_{a}} T\left[\gamma^{(2)} \begin{array}{rrr}x & y & z \\ \zeta_{x} \zeta_{y} \zeta_{z}\end{array}\right]=2 \zeta_{a}\left\langle\Psi_{\zeta_{x}^{2} \zeta_{y}^{2} \zeta_{z}^{2}}\left[\gamma^{(2)}\right]\left|\hat{T}_{a}\right| \Psi_{\zeta_{x}^{2} \zeta_{y}^{2} \zeta_{z}^{2}}\left[\gamma^{(2)}\right]\right\rangle_{\Omega}$

The proof of Eq. (27) is given in Appendix C. Similarly, the derivative of both sides of Eq. (26) with respect to $\xi$ leads to

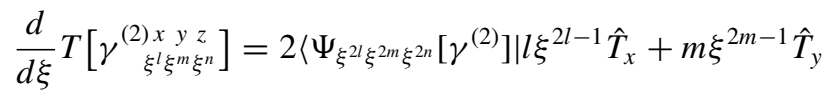

$$
\begin{aligned}
& +n \xi^{2 n-1} \hat{T}_{z} \mid \Psi_{\left.\xi^{2 l} \xi^{2 m} \xi^{2 n}\left[\gamma^{(2)}\right]\right\rangle_{\Omega} .}
\end{aligned}
$$

Equation (28) is proven in the same way as the proof in Appendix C.

Equations (25)-(28) are fundamental properties of the $\mathrm{KE}$ functional. In the subsequent Sec. III A 2, using these properties, we derive the exact relations and bounds for the KE functional.

\section{Derivations of the exact relations and bounds for the KE functional}

From Eq. (25), $\quad T\left[\begin{array}{rrrr}(2) & x & y & z \\ \zeta_{x} & \zeta_{y} & \zeta_{z}\end{array}\right]$ is equal to

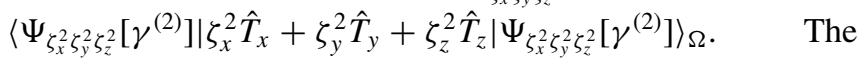
expectation value of $\zeta_{x}^{2} \hat{T}_{x}+\zeta_{y}^{2} \hat{T}_{y}+\zeta_{z}^{2} \hat{T}_{z}$ with respect to $\left|\Psi\left[\gamma^{(2)}\right]\right\rangle$ is equal to or larger than the expectation value with respect to $\left|\Psi_{\zeta_{x}^{2} \zeta_{y}^{2} \zeta_{z}^{2}}\left[\gamma^{(2)}\right]\right\rangle$, since $\left|\Psi_{\zeta_{x}^{2} \zeta_{y}^{2} \zeta_{z}^{2}}\left[\gamma^{(2)}\right]\right\rangle$ is the minimizing wave function. Thus, we have the following inequality:

$$
T\left[\gamma^{(2)} \begin{array}{rrr}
x & y & z \\
\zeta_{x} \zeta_{y} \zeta_{z}
\end{array}\right] \leqslant\left\langle\Psi\left[\gamma^{(2)}\right]\left|\zeta_{x}^{2} \hat{T}_{x}+\zeta_{y}^{2} \hat{T}_{y}+\zeta_{z}^{2} \hat{T}_{z}\right| \Psi\left[\gamma^{(2)}\right]\right\rangle_{\Omega} .
$$

Substituting Eq. (27) into the right-hand side of Eq. (29), we get

$$
\begin{aligned}
& T\left[\gamma^{(2)} \begin{array}{rrr}
x & y & z \\
\zeta_{x} \zeta_{y} \zeta_{z}
\end{array}\right] \leqslant \frac{\zeta_{x}^{2}}{2}\left\{\frac{d T\left[\begin{array}{rrr}
(2) & x & y \\
\zeta_{x} & 1 & 1
\end{array}\right]}{d \zeta_{x}}\right\}_{\zeta_{x}=1}
\end{aligned}
$$

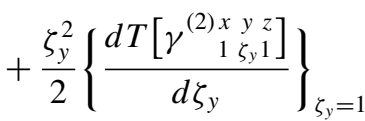

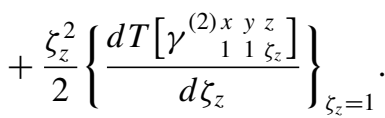

This inequality is one of the exact relations that should be satisfied with the KE functional. 
The other relations can be obtained by using Eq. (27). Multiplying both sides of Eq. (27) by $\zeta_{a}^{\delta-1}$, we have

$$
\frac{\zeta_{a}^{\delta-1}}{2} \frac{d T\left[\begin{array}{ccc}
(2) x & y & z \\
\zeta_{x} \zeta_{y} \zeta_{z}
\end{array}\right]}{d \zeta_{a}}=\left\langle\Psi_{\zeta_{x}^{2} \zeta_{y}^{2} \zeta_{z}^{2}}\left[\gamma^{(2)}\right]\left|\zeta_{a}^{\delta} \hat{T}_{a}\right| \Psi_{\zeta_{x}^{2} \zeta_{y}^{2} \zeta_{z}^{2}}\left[\gamma^{(2)}\right]\right\rangle_{\Omega} \quad(a=x, y, z)
$$

where $\delta$ is an arbitrary real number. Summing the cases when $a=x, y, z$ of Eq. (31), we have

$$
\begin{aligned}
& \frac{\zeta_{x}^{\delta-1}}{2} \frac{d T\left[\begin{array}{ccc}
(2) x & y & z \\
\zeta_{x} \zeta_{y} \zeta_{z}
\end{array}\right]}{d \zeta_{x}}+\frac{\zeta_{y}^{\delta-1}}{2} \frac{d T\left[\begin{array}{rrr}
(2) x & y & z \\
\zeta_{x} \zeta_{y} \zeta_{z}
\end{array}\right]}{d \zeta_{y}}+\frac{\zeta_{z}^{\delta-1}}{2} \frac{d T\left[\begin{array}{ccc}
(2) x & y & z \\
\zeta_{x} \zeta_{y} \zeta_{z}
\end{array}\right]}{d \zeta_{z}} \\
& =\left\langle\Psi_{\zeta_{x}^{2} \zeta_{y}^{2} \zeta_{z}^{2}}\left[\gamma^{(2)}\right]\left|\zeta_{x}^{\delta} \hat{T}_{x}+\zeta_{y}^{\delta} \hat{T}_{y}+\zeta_{z}^{\delta} \hat{T}_{z}\right| \Psi_{\zeta_{x}^{2} \zeta_{y}^{2} \zeta_{z}^{2}}\left[\gamma^{(2)}\right]\right\rangle_{\Omega}
\end{aligned}
$$

From the definition of $\left|\Psi_{\zeta_{x}^{\delta} \zeta_{y}^{\delta} \zeta_{z}^{\delta}}\left[\gamma^{(2)}\right]\right\rangle$, the right-hand side of Eq. (32) is equal to or more than $\left\langle\Psi_{\zeta_{x}^{\delta} \zeta_{y}^{\delta} \zeta_{z}^{\delta}}\left[\gamma^{(2)}\right]\right| \zeta_{x}^{\delta} \hat{T}_{x}+\zeta_{y}^{\delta} \hat{T}_{y}+$

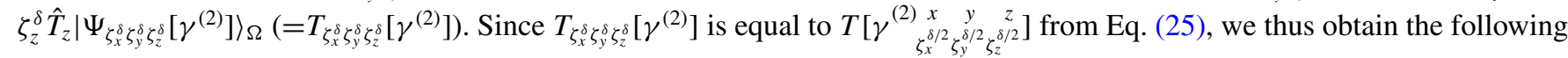
inequality;

$$
\frac{\zeta_{x}^{\delta-1}}{2} \frac{d T\left[\begin{array}{rrr}
(2) x & y & z \\
\zeta_{x} \zeta_{y} \zeta_{z}
\end{array}\right]}{d \zeta_{x}}+\frac{\zeta_{y}^{\delta-1}}{2} \frac{d T\left[\begin{array}{rrr}
(2) x & y & z \\
\zeta_{x} \zeta_{y} \zeta_{z}
\end{array}\right]}{d \zeta_{y}}+\frac{\zeta_{z}^{\delta-1}}{2} \frac{d T\left[\begin{array}{rrr}
(2) x & y & z \\
\zeta_{x} \zeta_{y} \zeta_{z}
\end{array}\right]}{d \zeta_{z}} \geqslant T\left[\begin{array}{cccc}
(2) & x & y & z \\
\zeta_{x}^{\delta / 2} & \zeta_{y}^{\delta / 2} & \zeta_{z}^{\delta / 2}
\end{array}\right],
$$

where the equal sign holds when $\delta$ is equal to 2. Equation (33) is also one of exact relations for the KE functional. It should be noted that if we consider an isolated system, Eq. (33) with $\delta=2$ is reduced to

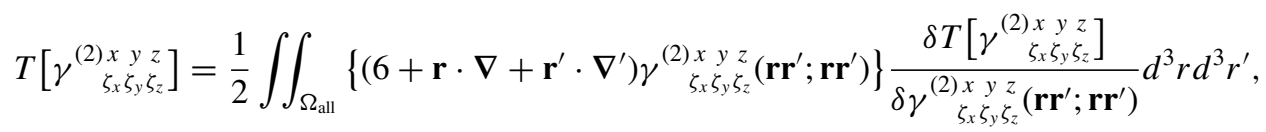

where $\Omega_{\text {all }}$ denotes the whole space. We may soon find that Eq. (34) coincides with the relation that is derived by means of the uniform coordinate scaling of electrons [24]. This means that Eq. (33) includes the previous results [24] and may be useful for further developments of the approximate KE functional.

By using Eqs. (26) and (28), we also obtain the other relation for the KE functional. Multiplying both sides of Eq. (28) by $\xi$, we have

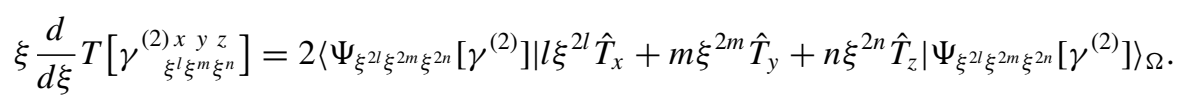

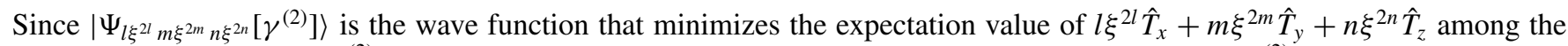
wave functions that yield $\gamma^{(2)}$, the right-hand side of Eq. (35) is equal to or more than $2 T_{l \xi^{2 l}} m \xi^{2 m} n \xi^{2 n}\left[\gamma^{(2)}\right]$. If the values of $l, m$,

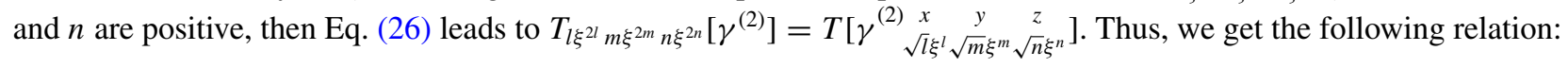

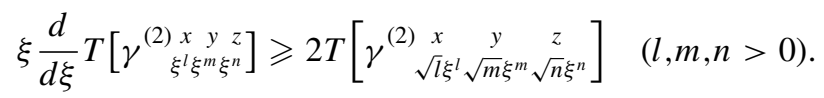

This relation is also one of exact relations that should be fulfilled by the KE functional.

Equations (30), (33), and (36) are regarded as exact relations for the KE functional. These relations, as well as the previously derived relations [24], can be utilized in evaluating and/or developing the approximate forms of the KE functional of the PD-functional theory. In Sec. III C, we show examples of using Eqs. (30), (33), and (36) as restrictive conditions on the KE functional.

\section{Illustrative examples}

Equations (30), (33), and (36) have a general form, so that one can make diverse choices of scaling factors $\left(\zeta_{x}, \zeta_{y}, \zeta_{z}\right.$, and $\xi)$ and parameters $(\delta, l, m$, and $n)$. In this subsubsection, we show illustrative examples of the exact relations and bounds.
Let us consider the specific nonuniform coordinate scaling such that $\zeta_{x}=\zeta, \zeta_{y}=\zeta_{z}=1$. In this case, the lower bounds for $T\left[\begin{array}{lll}\gamma^{(2)} \times & y & z \\ \zeta & 1\end{array}\right]$ can be obtained by summing the cases when $\delta=$

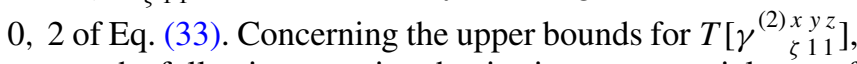
we use the following equation that is given as a special case of Eq. (33) $\left(\delta=2, \zeta_{x}=\zeta_{y}=\zeta_{z}=1\right)$ :

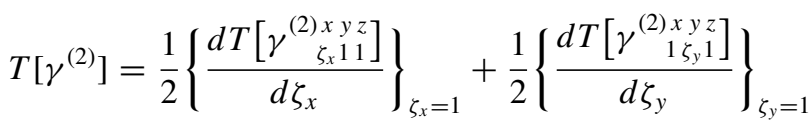

$$
\begin{aligned}
& +\frac{1}{2}\left\{\frac{d T\left[\gamma^{(2) x y z} \begin{array}{c}
11 \zeta_{z}
\end{array}\right]}{d \zeta_{z}}\right\}_{\zeta_{z}=1} .
\end{aligned}
$$


Substituting Eq. (37) into the right-hand side of Eq. (30), we get the upper bounds for $T\left[\gamma_{\zeta 11}^{(2) x y z}\right.$. Thus, we have

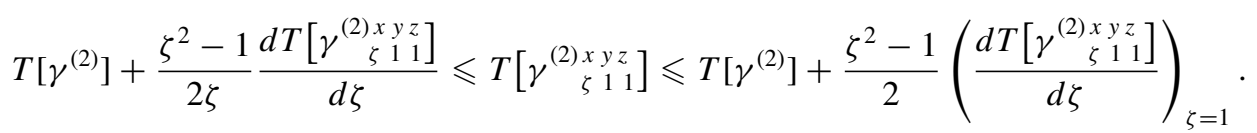

If we consider the specific case when $\zeta_{x}=\zeta_{y}=\zeta, \zeta_{z}=1$, then the upper and lower bounds for $T\left[\begin{array}{r}\gamma^{(2) x y z} \\ \zeta \zeta 1\end{array}\right]$ are also derived from Eqs. (30) and (33) in a way similar to Eq. (38). We have

$$
T\left[\gamma^{(2)}\right]+\frac{\zeta^{2}-1}{2 \zeta} \frac{d T\left[\begin{array}{r}
(2) x y z \\
\zeta \zeta 1
\end{array}\right]}{d \zeta} \leqslant T\left[\gamma_{\zeta \zeta 1}^{(2) x y z}\right] \leqslant T\left[\gamma^{(2)}\right]+\frac{\zeta^{2}-1}{2}\left(\frac{d T\left[\begin{array}{r}
(2) x y z \\
\zeta \zeta 1
\end{array}\right]}{d \zeta}\right)_{\zeta=1} .
$$

Thus, exact relations obtained in Sec. III A 2 are versatile, and they could include various special cases.

\section{B. Invariance of the KE functional under the translation and rotation}

In the conventional DFT, the translation and rotation of electron coordinates have already been discussed in deriving the relations for the xc energy functional [78]. In this subsection, we derive the relation for the $\mathrm{KE}$ functional by discussing how the KE functional changes under the translation and rotation of electron coordinates.

Let us consider the transformation such that $\mathbf{r}_{i}$ is transformed into $\mathbf{r}_{i}^{\prime}=R^{-1}\left(\mathbf{r}_{i}-\mathbf{a}\right)$, where a and $R^{-1}$ denote a translation vector and the inversion of a rotation matrix $R$, respectively. If $\mathbf{r}_{i}$ varies in a volume $\Omega$, then we denote the domain of $\mathbf{r}_{i}^{\prime}$ by $\Omega_{R, \mathbf{a}}$. By this transformation, the wave function $\Psi\left(\mathbf{r}_{1}, \ldots, \mathbf{r}_{N}, \eta_{1}, \ldots, \eta_{N}\right)$ is transformed into

$$
\begin{aligned}
\Psi_{R, \mathbf{a}}\left(\mathbf{r}_{1}, \ldots, \mathbf{r}_{N}, \eta_{1}, \ldots, \eta_{N}\right)= & \Psi\left(R \mathbf{r}_{1}+\mathbf{a}, \ldots, R \mathbf{r}_{N}\right. \\
& \left.+\mathbf{a}, \eta_{1}, \ldots, \eta_{N}\right),
\end{aligned}
$$

where we refer to $\Psi_{R, \mathbf{a}}\left(\mathbf{r}_{1}, \ldots, \mathbf{r}_{N}, \eta_{1}, \ldots, \eta_{N}\right)$ as the transformed wave function. Note that the transformed wave function is normalized to unity in the volume $\Omega_{R, \mathbf{a}}$. The PD that is calculated from Eq. (40) is given by

$\gamma_{R, \mathbf{a}}^{(2)}\left(\mathbf{r r}^{\prime} ; \mathbf{r r}^{\prime}\right)=\gamma^{(2)}\left(R \mathbf{r}+\mathbf{a} R \mathbf{r}^{\prime}+\mathbf{a} ; R \mathbf{r}+\mathbf{a} R \mathbf{r}^{\prime}+\mathbf{a}\right)$.

It can be shown that $\Psi\left[\gamma_{R, \mathbf{a}}^{(2)}\right]\left(\mathbf{r}_{1}, \ldots, \mathbf{r}_{N}, \eta_{1}, \ldots, \eta_{N}\right)$ is equal to $\Psi\left[\gamma^{(2)}\right]\left(R \mathbf{r}_{1}+\mathbf{a}, \ldots, R \mathbf{r}_{N}+\mathbf{a}, \eta_{1}, \ldots, \eta_{N}\right)$ by comparing the equation for $\Psi\left[\gamma_{R, \mathbf{a}}^{(2)}\right]\left(\mathbf{r}_{1}, \ldots, \mathbf{r}_{N}, \eta_{1}, \ldots, \eta_{N}\right)$ with that for $\Psi\left[\gamma^{(2)}\right]\left(R \mathbf{r}_{1}+\mathbf{a}, \ldots, R \mathbf{r}_{N}+\mathbf{a}, \eta_{1}, \ldots, \eta_{N}\right)$. The proof is similar to that given in Appendix B. This equality immediately leads to

$$
T\left[\gamma^{(2)}\right]=T\left[\gamma_{R, \mathbf{a}}^{(2)}\right] .
$$

Equation (42) can be utilized in evaluating and/or developing approximate forms of the KE functional. Examples of using Eq. (42) are shown in Sec. III C.

\section{Examples of using exact relations and bounds}

In a previous paper [24], we have proposed the approximate functional that fulfils the requirement that has been derived by means of the Hohenberg-Kohn theorem of the PD. The KE functional is given by [24]

$$
T\left[\gamma^{(2)}\right]=\iint f\left(\mathbf{r}, \mathbf{r}^{\prime}\right) \gamma^{(2)}\left(\mathbf{r r}^{\prime} ; \mathbf{r r}^{\prime}\right) d^{3} r d^{3} r^{\prime},
$$

where $f\left(\mathbf{r}, \mathbf{r}^{\prime}\right)$ is a function that should be determined from the other relations for the KE functional. In the previous works, $f\left(\mathbf{r}, \mathbf{r}^{\prime}\right)$ is determined by requiring it to satisfy the relation that is derived via the uniform coordinate scaling of electrons [24]. The condition on $f\left(\mathbf{r}, \mathbf{r}^{\prime}\right)$ is given by [24]

$$
\begin{aligned}
& \iint\left\{2 f\left(\mathbf{r}, \mathbf{r}^{\prime}\right)+\mathbf{r} \cdot \nabla f\left(\mathbf{r}, \mathbf{r}^{\prime}\right)+\mathbf{r}^{\prime} \cdot \nabla^{\prime} f\left(\mathbf{r}, \mathbf{r}^{\prime}\right)\right\} \\
& \quad \times \gamma^{(2)}\left(\mathbf{r} \mathbf{r}^{\prime} ; \mathbf{r r}^{\prime}\right) d^{3} r d^{3} r^{\prime}=0 .
\end{aligned}
$$

Instead of the above relation, if we impose Eqs. (30), (33), (36), and (42) on Eq. (43), then $f\left(\mathbf{r}, \mathbf{r}^{\prime}\right)$ should fulfill the following conditions;

$$
\begin{aligned}
& \iint\left[\frac{\zeta_{x}^{2}}{2}\left\{\frac{d f\left(\frac{x}{\zeta_{x}}, y, z, \frac{x^{\prime}}{\zeta_{x}}, y^{\prime}, z^{\prime}\right)}{d \zeta_{x}}\right\}_{\zeta_{x}=1}+\frac{\zeta_{y}^{2}}{2}\left\{\frac{d f\left(x, \frac{y}{\zeta_{y}}, z, x^{\prime}, \frac{y^{\prime}}{\zeta_{y}}, z^{\prime}\right)}{d \zeta_{y}}\right\}_{\zeta_{y}=1}\right. \\
& \left.\quad+\frac{\zeta_{z}^{2}}{2}\left\{\frac{d f\left(x, y, \frac{z}{\zeta_{z}}, x^{\prime}, y^{\prime}, \frac{z^{\prime}}{\zeta_{z}}\right)}{d \zeta_{z}}\right\}_{\zeta_{z}=1}-f\left(\frac{x}{\zeta_{x}}, \frac{y}{\zeta_{y}}, \frac{z}{\zeta_{z}}, \frac{x^{\prime}}{\zeta_{x}}, \frac{y^{\prime}}{\zeta_{y}}, \frac{z^{\prime}}{\zeta_{z}}\right)\right] \gamma^{(2)}\left(\mathbf{r r}^{\prime} ; \mathbf{r r}^{\prime}\right) d^{3} r d^{3} r^{\prime} \geqslant 0,
\end{aligned}
$$




$$
\begin{aligned}
& \iint {\left[\left(\frac{\zeta_{x}^{\delta-1}}{2} \frac{\partial}{\partial \zeta_{x}}+\frac{\zeta_{y}^{\delta-1}}{2} \frac{\partial}{\partial \zeta_{y}}+\frac{\zeta_{z}^{\delta-1}}{2} \frac{\partial}{\partial \zeta_{z}}\right) f\left(\frac{x}{\zeta_{x}}, \frac{y}{\zeta_{y}}, \frac{z}{\zeta_{z}}, \frac{x^{\prime}}{\zeta_{x}}, \frac{y^{\prime}}{\zeta_{y}}, \frac{z^{\prime}}{\zeta_{z}}\right)\right.} \\
&\left.-f\left(\frac{x}{\zeta_{x}^{\delta / 2}}, \frac{y}{\zeta_{y}^{\delta / 2}}, \frac{z}{\zeta_{z}^{\delta / 2}}, \frac{x^{\prime}}{\zeta_{x}^{\delta / 2}}, \frac{y^{\prime}}{\zeta_{y}^{\delta / 2}}, \frac{z^{\prime}}{\zeta_{z}^{\delta / 2}}\right)\right] \gamma^{(2)}\left(\mathbf{r r}^{\prime} ; \mathbf{r r}^{\prime}\right) d^{3} r d^{3} r^{\prime} \geqslant 0 \\
& \iint\left[\xi \frac{d}{d \xi} f\left(\frac{x}{\xi^{l}}, \frac{y}{\xi^{m}}, \frac{z}{\xi^{n}}, \frac{x^{\prime}}{\xi^{l}}, \frac{y^{\prime}}{\xi^{m}}, \frac{z^{\prime}}{\xi^{n}}\right)-2 f\left(\frac{x}{\sqrt{l} \xi^{l}}, \frac{y}{\sqrt{m} \xi^{m}}, \frac{z}{\sqrt{n} \xi^{n}}, \frac{x^{\prime}}{\sqrt{l} \xi^{l}}, \frac{y^{\prime}}{\sqrt{m} \xi^{m}}, \frac{z^{\prime}}{\sqrt{n} \xi^{n}}\right)\right] \gamma^{(2)}\left(\mathbf{r r}^{\prime} ; \mathbf{r r}^{\prime}\right) d^{3} r d^{3} r^{\prime} \geqslant 0, \\
& \iint\left\{f\left(\mathbf{r}, \mathbf{r}^{\prime}\right)-f\left(R^{-1}(\mathbf{r}-\mathbf{a}), R^{-1}\left(\mathbf{r}^{\prime}-\mathbf{a}\right)\right)\right\} \gamma^{(2)}\left(\mathbf{r r}^{\prime} ; \mathbf{r r}^{\prime}\right) d^{3} r d^{3} r^{\prime}=0 \\
&\left.\iint \frac{(48)}{(48)}\right)
\end{aligned}
$$

respectively, and where $f\left(\mathbf{r}, \mathbf{r}^{\prime}\right)$ is rewritten as $f\left(x, y, z, x^{\prime}, y^{\prime}, z^{\prime}\right)$ in Eqs. (45)-(47). Furthermore, as the sufficient condition for satisfying the above conditions, we respectively obtain

$$
\begin{gathered}
\frac{\zeta_{x}^{2}}{2}\left\{\frac{d f\left(\frac{x}{\zeta_{x}}, y, z, \frac{x^{\prime}}{\zeta_{x}}, y^{\prime}, z^{\prime}\right)}{d \zeta_{x}}\right\}_{\zeta_{x}=1}+\frac{\zeta_{y}^{2}}{2}\left\{\frac{d f\left(x, \frac{y}{\zeta_{y}}, z, x^{\prime}, \frac{y^{\prime}}{\zeta_{y}}, z^{\prime}\right)}{d \zeta_{y}}\right\}_{\zeta_{y}=1} \\
+\frac{\zeta_{z}^{2}}{2}\left\{\frac{d f\left(x, y, \frac{z}{\zeta_{z}}, x^{\prime}, y^{\prime}, \frac{z^{\prime}}{\zeta_{z}}\right)}{d \zeta_{z}}\right\}_{\zeta_{z}=1}-f\left(\frac{x}{\zeta_{x}}, \frac{y}{\zeta_{y}}, \frac{z}{\zeta_{z}}, \frac{x^{\prime}}{\zeta_{x}}, \frac{y^{\prime}}{\zeta_{y}}, \frac{z^{\prime}}{\zeta_{z}}\right) \geqslant 0 \\
\left(\frac{\zeta_{x}^{\delta-1}}{2} \frac{\partial}{\partial \zeta_{x}}+\frac{\zeta_{y}^{\delta-1}}{2} \frac{\partial}{\partial \zeta_{y}}+\frac{\zeta_{z}^{\delta-1}}{2} \frac{\partial}{\partial \zeta_{z}}\right) f\left(\frac{x}{\zeta_{x}}, \frac{y}{\zeta_{y}}, \frac{z}{\zeta_{z}}, \frac{x^{\prime}}{\zeta_{x}}, \frac{y^{\prime}}{\zeta_{y}}, \frac{z^{\prime}}{\zeta_{z}}\right)-f\left(\frac{x}{\zeta_{x}^{\delta / 2}}, \frac{y}{\zeta_{y}^{\delta / 2}}, \frac{z}{\zeta_{z}^{\delta / 2}}, \frac{x^{\prime}}{\zeta_{x}^{\delta / 2}}, \frac{y^{\prime}}{\zeta_{y}^{\delta / 2}}, \frac{z^{\prime}}{\zeta_{z}^{\delta / 2}}\right) \geqslant 0, \\
\xi_{\frac{d}{d \xi}} f\left(\frac{x}{\xi^{l}}, \frac{y}{\xi^{m}}, \frac{z}{\xi^{n}}, \frac{x^{\prime}}{\xi^{l}}, \frac{y^{\prime}}{\xi^{m}}, \frac{z^{\prime}}{\xi^{n}}\right)-2 f\left(\frac{x}{\sqrt{l} \xi^{l}}, \frac{y}{\sqrt{m} \xi^{m}}, \frac{z}{\sqrt{n} \xi^{n}}, \frac{x^{\prime}}{\sqrt{l} \xi^{l}}, \frac{y^{\prime}}{\sqrt{m} \xi^{m}}, \frac{z^{\prime}}{\sqrt{n} \xi^{n}}\right) \geqslant 0 \\
f\left(\mathbf{r}, \mathbf{r}^{\prime}\right)=f\left(R^{-1}(\mathbf{r}-\mathbf{a}), R^{-1}\left(\mathbf{r}^{\prime}-\mathbf{a}\right)\right) .
\end{gathered}
$$

If $f\left(\mathbf{r}, \mathbf{r}^{\prime}\right)$ fulfils Eqs. (49)-(52), then Eq. (43) satisfies exact relations Eqs. (30), (33), (36), and (42), respectively.

We apply Eqs. (49)-(52) to checking the validity of the following KE functionals that have previously been proposed by using the condition Eq. (44) [24]:

$$
\begin{aligned}
& \text { (i) } \iint \frac{K}{\left|\mathbf{r}-\mathbf{r}^{\prime}\right|^{2}} \gamma^{(2)}\left(\mathbf{r} \mathbf{r}^{\prime} ; \mathbf{r r}^{\prime}\right) d^{3} r d^{3} r^{\prime}, \\
& \text { (ii) } \iint\left(\frac{K}{r^{2}}+\frac{K}{r^{\prime 2}}\right) \gamma^{(2)}\left(\mathbf{r} \mathbf{r}^{\prime} ; \mathbf{r r}^{\prime}\right) d^{3} r d^{3} r^{\prime}, \\
& \text { (ii) } \iint \frac{K}{\left|\mathbf{r}+\mathbf{r}^{\prime}\right|^{2}} \gamma^{(2)}\left(\mathbf{r} \mathbf{r}^{\prime} ; \mathbf{r} \mathbf{r}^{\prime}\right) d^{3} r d^{3} r^{\prime},
\end{aligned}
$$

where $K$ denotes a constant. Approximate forms (i), (ii), and (iii) correspond to the cases of $f\left(\mathbf{r}, \mathbf{r}^{\prime}\right)=K /\left|\mathbf{r}-\mathbf{r}^{\prime}\right|^{2}$, $K\left(1 / r^{2}+1 / r^{\prime 2}\right)$, and $K /\left|\mathbf{r}+\mathbf{r}^{\prime}\right|^{2}$, respectively. Results of the evaluations are summarized in Table I. Judging from the number of satisfactory relations and bounds, it is expected that the approximate form (i) shows good behavior in comparison with the others. Also, when making the approximate forms better and when proposing the other new functionals,
Eqs. (49)-(52) may work as the requirements. Thus, exact relations and bounds obtained in Secs. III A and III B can be certainly used in developing the approximate form of $T\left[\gamma^{(2)}\right]$.

\section{CONCLUDING REMARKS}

The development of the approximate form of the KE functional is one of the main issues in the PD-functional theory. We address this issue with two strategies by reference to

TABLE I. Evaluations of approximate forms of the KE functional by means of Eqs. (49)-(52)

\section{(i)}

(ii)

(iii)

\begin{tabular}{llll}
\hline Eq. (49) & Yes & Yes & Yes \\
Eq. (50) & Yes & Yes & Yes \\
Eq. (51) & Yes & Yes & Yes \\
Eq. (52) & Yes & No & No \\
\hline \hline
\end{tabular}


the established method to develop the approximate xc energy functional of the DFT. The first strategy is to devise the approximate functional on the basis of the coupling-constant expression for the KE functional. In this paper, we derive the coupling-constant expression and show that it serves as a sound foundation to develop approximate forms of the KE functional.

The second strategy is to develop the approximate functional by utilizing relations and bounds for the KE functional as restrictive conditions. In this paper, three kinds of exact relations are obtained successfully by investigating nonuniform scaling properties of the KE functional. On the basis of such exact relations, we illustrate a few examples of upper and lower bounds for the KE functional. Also, we show examples of how the exact relations are used as restrictive conditions. Thus, the relations and bounds obtained here become strong restrictive conditions in developing and/or evaluating the approximate form of the KE functional. In addition, it is shown in this paper that the KE functional does not change under the rotation and translation of electron coordinates. This fact also becomes a useful restrictive condition on the approximate form of the KE functional as shown in Sec. III C.

Thus, the two abovementioned strategies are considered to provide promising approaches to develop the approximate KE functional of the PD-functional theory.

\section{ACKNOWLEDGMENT}

This work was partially supported by Grants-in-Aid for Scientific Research (No. 22540390 and No. 23540446) of the Ministry of Education, Culture, Sports, Science, and Technology, Japan.

\section{APPENDIX A: NOTATIONS USED IN SEC. III A}

In this Appendix, we summarize the notations that are used in Sec. III A.

$\zeta_{x}, \zeta_{y}$, and $\zeta_{z}$. These denote scale factors of the nonuniform coordinate scaling of electrons such that $\left(x_{i}, y_{i}, z_{i}\right) \rightarrow$ $\left(\zeta_{x}^{-1} x_{i}, \zeta_{y}^{-1} y_{i}, \zeta_{z}^{-1} z_{i}\right)$

$\Psi_{\zeta_{x} \zeta_{y} \zeta_{z}}^{x} \zeta_{z}\left(\mathbf{r}_{1}, \ldots, \mathbf{r}_{N}, \eta_{1}, \ldots, \eta_{N}\right)$. This is the scaled wave function that is transformed from $\Psi\left(\mathbf{r}_{1}, \ldots, \mathbf{r}_{N}, \eta_{1}, \ldots, \eta_{N}\right)$ by the nonuniform coordinate scaling of electrons such that $\left(x_{i}, y_{i}, z_{i}\right) \rightarrow\left(\zeta_{x}^{-1} x_{i}, \zeta_{y}^{-1} y_{i}, \zeta_{z}^{-1} z_{i}\right)$. The definition is given in Eq. (16).

$\Psi\left[\gamma^{(2)}\right]_{\zeta_{x} \zeta_{y} \zeta_{z}}^{x} y_{z}\left(\mathbf{r}_{1}, \ldots, \mathbf{r}_{N}, \eta_{1}, \ldots, \eta_{N}\right) . \quad$ This is the scaled wave function that is transformed from $\Psi\left[\gamma^{(2)}\right]\left(\mathbf{r}_{1}, \ldots, \mathbf{r}_{N}, \eta_{1}, \ldots, \eta_{N}\right)$ by the nonuniform coordinate scaling of electrons such that $\left(x_{i}, y_{i}, z_{i}\right) \rightarrow$ $\left(\zeta_{x}^{-1} x_{i}, \zeta_{y}^{-1} y_{i}, \zeta_{z}^{-1} z_{i}\right)$

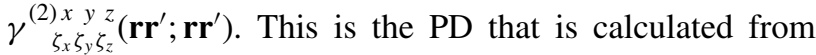
$\Psi_{\zeta_{x} \zeta_{y} \zeta_{z}}^{x} \begin{gathered}z \\ z\end{gathered}\left(\mathbf{r}_{1}, \ldots, \mathbf{r}_{N}, \eta_{1}, \ldots, \eta_{N}\right)$. The definition is given in Eq. (17).

$\alpha, \beta$, and $\gamma$. These denote the factors that enlarge or reduce the KE operator anisotropically such as $\alpha \hat{T}_{x}+\beta \hat{T}_{y}+\gamma \hat{T}_{z}$.

$T_{\alpha \beta \gamma}\left[\gamma^{(2)}\right]$. This is the modified KE functional that is enlarged or reduced anisotropically by the factors $\alpha, \beta$, and $\gamma$. The definition is given in Eq. (18).

$\Psi_{\alpha \beta \gamma}\left[\gamma^{(2)}\right]\left(\mathbf{r}_{1}, \ldots, \mathbf{r}_{N}, \eta_{1}, \ldots, \eta_{N}\right)$. This wave function minimizes the expectation value of $\alpha \hat{T}_{x}+\beta \hat{T}_{y}+\gamma \hat{T}_{z}$ among antisymmetric wave functions that yield $\gamma^{(2)}\left(\mathbf{r r}^{\prime} ; \mathbf{r r}^{\prime}\right)$. The definition is given in the second line of Eq. (18).

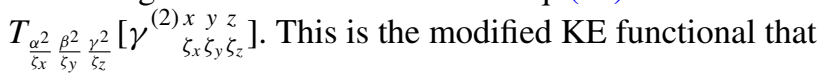
is enlarged or reduced anisotropically by the factors $\alpha^{2} / \zeta_{x}$, $\beta^{2} / \zeta_{y}$, and $\gamma^{2} / \zeta_{z}$. The definition is given in Eq. (20).

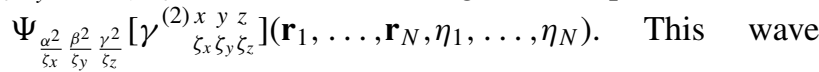
function minimizes the expectation value of $\frac{\alpha^{2}}{\zeta_{x}} \hat{T}_{x}+\frac{\beta^{2}}{\zeta_{y}} \hat{T}_{y}+$ $\frac{\gamma^{2}}{\zeta_{z}}$ among antisymmetric wave functions that yield $\gamma_{\zeta_{x} \zeta_{y} \zeta_{z}}^{\zeta_{z}}\left(\mathbf{r r}^{\prime} ; \mathbf{r r}^{\prime}\right)$. The definition is given in the second line of Eq. (20).

$\Psi_{\alpha \beta \gamma}\left[\gamma^{(2)}\right]_{\zeta_{x} \zeta_{y} \zeta_{z}}^{x y z}\left(\mathbf{r}_{1}, \ldots, \mathbf{r}_{N}, \eta_{1}, \ldots, \eta_{N}\right)$. This is the scaled wave function that is transformed from $\Psi_{\alpha \beta \gamma}\left[\gamma^{(2)}\right]$ $\left(\mathbf{r}_{1}, \ldots, \mathbf{r}_{N}, \eta_{1}, \ldots, \eta_{N}\right)$ by the nonuniform coordinate scaling of electrons such that $\left(x_{i}, y_{i}, z_{i}\right) \rightarrow$ $\left(\zeta_{x}^{-1} x_{i}, \zeta_{y}^{-1} y_{i}, \zeta_{z}^{-1} z_{i}\right)$. The definition is given in Eq. (22).

\section{APPENDIX B: PROOF OF EQ. (21)}

In this Appendix, we prove Eq. (21). Since $\left|\Psi_{\alpha \beta \gamma}\left[\gamma^{(2)}\right]\right\rangle$ is defined in Eq. (18) as the minimizing wave function that yields a prescribed PD $\gamma^{(2)}\left(\mathbf{r r}^{\prime} ; \mathbf{r r}^{\prime}\right)$ and is normalized to unity, $\left|\Psi_{\alpha \beta \gamma}\left[\gamma^{(2)}\right]\right\rangle$ obeys the following equation;

$$
\left\{\alpha \hat{T}_{x}+\beta \hat{T}_{y}+\gamma \hat{T}_{z}+\iint_{\Omega} \mu_{\alpha \beta \gamma}\left[\gamma^{(2)}\right]\left(\mathbf{r}, \mathbf{r}^{\prime}\right) \hat{\gamma}^{(2)}\left(\mathbf{r r}^{\prime} ; \mathbf{r r}^{\prime}\right) d^{3} r d^{3} r^{\prime}\right\}\left|\Psi_{\alpha \beta \gamma}\left[\gamma^{(2)}\right]\right\rangle=\varepsilon_{\alpha \beta \gamma}\left[\gamma^{(2)}\right]\left|\Psi_{\alpha \beta \gamma}\left[\gamma^{(2)}\right]\right\rangle,
$$

where $\mu_{\alpha \beta \gamma}\left[\gamma^{(2)}\right]\left(\mathbf{r}, \mathbf{r}^{\prime}\right)$ and $\varepsilon_{\alpha \beta \gamma}\left[\gamma^{(2)}\right]$ are the Lagrange multipliers that correspond to the constraint such that $\left|\Psi_{\alpha \beta \gamma}\left[\gamma^{(2)}\right]\right\rangle$ yields a prescribed PD $\gamma^{(2)}\left(\mathbf{r r}^{\prime} ; \mathbf{r r}^{\prime}\right)$, and constraint on the normalization of $\left|\Psi_{\alpha \beta \gamma}\left[\gamma^{(2)}\right]\right\rangle$, respectively. By transforming the electron coordinates from $\mathbf{r}_{i}=\left(x_{i}, y_{i}, z_{i}\right)$ to $\mathbf{r}_{i}^{\prime}=\left(\zeta_{x}^{-1} x_{i}, \zeta_{y}^{-1} y_{i}, \zeta_{z}^{-1} z_{i}\right)$, Eq. (B1) is rewritten as

$$
\begin{aligned}
& \left\{\frac{\alpha}{\zeta_{x}^{2}} \hat{T}_{x}+\frac{\beta}{\zeta_{y}^{2}} \hat{T}_{y}+\frac{\gamma}{\zeta_{z}^{2}} \hat{T}_{z}+\iint_{\Omega^{\prime}} \mu_{\alpha \beta \gamma}\left[\gamma^{(2)}\right]\left(\zeta_{x} x, \zeta_{y} y, \zeta_{z} z, \zeta_{x} x^{\prime}, \zeta_{y} y^{\prime}, \zeta_{z} z^{\prime}\right) \hat{\gamma}^{(2)}\left(\mathbf{r r}^{\prime} ; \mathbf{r r}^{\prime}\right) d^{3} r d^{3} r^{\prime}\right\}\left|\Psi_{\alpha \beta \gamma}\left[\gamma^{(2)}\right]_{\zeta_{x} \zeta_{y} \zeta_{z}}^{x y}\right\rangle \\
& \quad=\varepsilon_{\alpha \beta \gamma}\left[\gamma^{(2)}\right]\left|\Psi_{\alpha \beta \gamma}\left[\gamma^{(2)}\right]_{\zeta_{x} \zeta_{y} \zeta_{z}}^{x y}\right\rangle .
\end{aligned}
$$




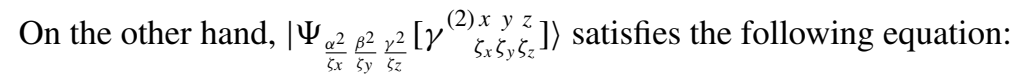

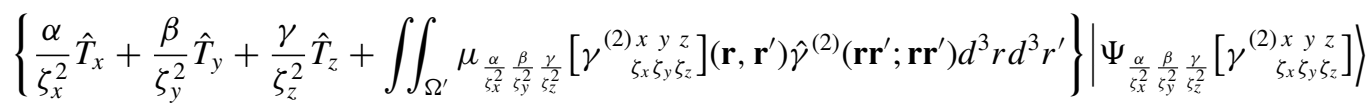

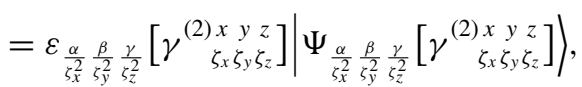

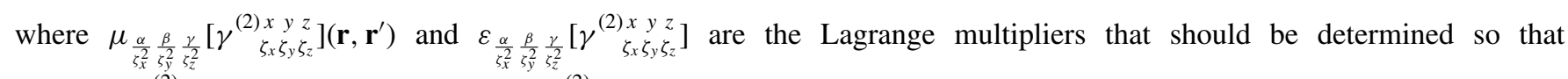

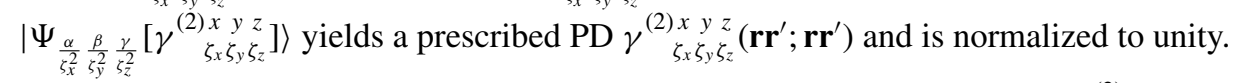

Let us consider the solution of Eq. (B3). We attempt to choose $\mu_{\alpha \beta \gamma}\left[\gamma^{(2)}\right]\left(\zeta_{x} x, \zeta_{y} y, \zeta_{z} z, \zeta_{x} x^{\prime}, \zeta_{y} y^{\prime}, \zeta_{z} z^{\prime}\right)$ and $\varepsilon_{\alpha \beta \gamma}\left[\gamma^{(2)}\right]$ as

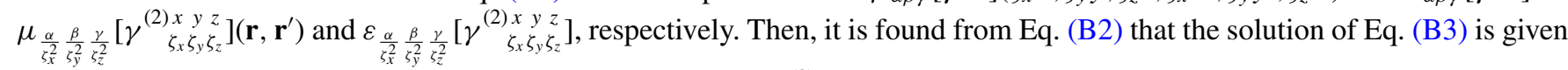
by $\left|\Psi_{\alpha \beta \gamma}\left[\gamma^{(2)}\right]_{\zeta_{x} \zeta_{y} \zeta_{z}}^{x y}\right\rangle$. Since $\left|\Psi_{\alpha \beta \gamma}\left[\gamma^{(2)}\right]_{\zeta_{x} \zeta_{y} \zeta_{z}}^{x y}\right\rangle$ actually yields $\left.\gamma_{\zeta_{x} \zeta_{y} \zeta_{z}}^{(2) x y} z \mathbf{r r}^{\prime} ; \mathbf{r r}^{\prime}\right)$ and is normalized to unity, the abovementioned choices of $\mu_{\frac{\alpha}{\zeta_{x}^{2}} \frac{\beta}{\zeta_{y}^{2}} \frac{\gamma}{\zeta_{z}^{2}}}\left[\gamma_{\zeta_{x} \zeta_{y} \zeta_{z}}^{(2) x y}\right]\left(\mathbf{r}, \mathbf{r}^{\prime}\right)$ and $\varepsilon_{\frac{\alpha}{\zeta_{x}^{2}} \xi_{y}^{\frac{\beta}{\zeta_{y}}} \frac{\gamma}{\zeta_{z}^{2}}}\left[\gamma_{\zeta_{x} \zeta_{y} \zeta_{z}}^{(2) x y} z\right]$ are correct. Thus, as the solution of Eq. (B3), we have

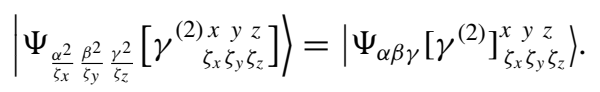

\section{APPENDIX C: PROOF OF EQ. (27)}

In this Appendix, we give the proof of Eq. (27) with $a=x$. From Eq. (26), we have

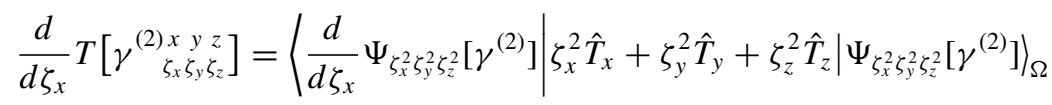

$$
\begin{aligned}
& +\left\langle\Psi_{\zeta_{x}^{2} \zeta_{y}^{2} \zeta_{z}^{2}}\left[\gamma^{(2)}\right]\left|\zeta_{x}^{2} \hat{T}_{x}+\zeta_{y}^{2} \hat{T}_{y}+\zeta_{z}^{2} \hat{T}_{z}\right| \frac{d}{d \zeta_{x}} \Psi_{\zeta_{x}^{2} \zeta_{y}^{2} \zeta_{z}^{2}}\left[\gamma^{(2)}\right]\right\rangle_{\Omega} \\
& +2 \zeta_{x}\left\langle\Psi_{\zeta_{x}^{2} \zeta_{y}^{2} \zeta_{z}^{2}}\left[\gamma^{(2)}\right]\left|\hat{T}_{x}\right| \Psi_{\zeta_{x}^{2} \zeta_{y}^{2} \zeta_{z}^{2}}\left[\gamma^{(2)}\right]\right\rangle_{\Omega} .
\end{aligned}
$$

Since $\left|\Psi_{\zeta_{x}^{2} \zeta_{y}^{2} \zeta_{z}^{2}}\left[\gamma^{(2)}\right]\right\rangle$ obeys Eq. (B1) with $\alpha=\zeta_{x}^{2}, \beta=\zeta_{y}^{2}$ and $\gamma=\zeta_{z}^{2}$, we get

$$
\varepsilon_{\zeta_{x}^{2} \zeta_{y}^{2} \zeta_{z}^{2}}\left[\gamma^{(2)}\right]=\left\langle\Psi_{\zeta_{x}^{2} \zeta_{y}^{2} \zeta_{z}^{2}}\left[\gamma^{(2)}\right]\left|\zeta_{x}^{2} \hat{T}_{x}+\zeta_{y}^{2} \hat{T}_{y}+\zeta_{z}^{2} \hat{T}_{z}+\iint_{\Omega} \mu_{\zeta_{x}^{2} \zeta_{y}^{2} \zeta_{z}^{2}}\left[\gamma^{(2)}\right]\left(\mathbf{r}, \mathbf{r}^{\prime}\right) \hat{\gamma}^{(2)}\left(\mathbf{r r}^{\prime} ; \mathbf{r r}^{\prime}\right) d^{3} r d^{3} r^{\prime}\right| \Psi_{\zeta_{x}^{2} \zeta_{y}^{2} \zeta_{z}^{2}}\left[\gamma^{(2)}\right]\right\rangle_{\Omega}
$$

where $\mu_{\zeta_{x}^{2} \zeta_{y}^{2} \zeta_{z}^{2}}\left[\gamma^{(2)}\right]\left(\mathbf{r}, \mathbf{r}^{\prime}\right)$ and $\varepsilon_{\zeta_{x}^{2} \zeta_{\zeta}^{2} \zeta_{z}^{2}}\left[\gamma^{(2)}\right]$ are the Lagrange multipliers that are determined from the constraints on $\left|\Psi_{\zeta_{x}^{2} \zeta_{y}^{2} \zeta_{z}^{2}}\left[\gamma^{(2)}\right]\right\rangle$. By differentiating both sides of Eq. (C2) with respect to $\zeta_{x}$, the Hellmann-Feynman theorem leads to

$$
\frac{d \varepsilon_{\zeta_{x}^{2} \zeta_{y}^{2} \zeta_{z}^{2}}\left[\gamma^{(2)}\right]}{d \zeta_{x}}=\left\langle\Psi_{\zeta_{x}^{2} \zeta_{y}^{2} \zeta_{z}^{2}}\left[\gamma^{(2)}\right]\left|2 \zeta_{x} \hat{T}_{x}+\iint_{\Omega} \frac{d \mu_{\zeta_{x}^{2} \zeta_{y}^{2} \zeta_{z}^{2}}\left[\gamma^{(2)}\right]\left(\mathbf{r}, \mathbf{r}^{\prime}\right)}{d \zeta_{x}} \hat{\gamma}^{(2)}\left(\mathbf{r} \mathbf{r}^{\prime} ; \mathbf{r r}^{\prime}\right) d^{3} r d^{3} r^{\prime}\right| \Psi_{\zeta_{x}^{2} \zeta_{y}^{2} \zeta_{z}^{2}}\left[\gamma^{(2)}\right]\right\rangle_{\Omega} .
$$

On the other hand, Eq. (C2) is rewritten by

$$
\varepsilon_{\zeta_{x}^{2} \zeta_{y}^{2} \zeta_{z}^{2}}\left[\gamma^{(2)}\right]=\left\langle\Psi_{\zeta_{x}^{2} \zeta_{y}^{2} \zeta_{z}^{2}}\left[\gamma^{(2)}\right]\left|\zeta_{x}^{2} \hat{T}_{x}+\zeta_{y}^{2} \hat{T}_{y}+\zeta_{z}^{2} \hat{T}_{z}\right| \Psi_{\zeta_{x}^{2} \zeta_{y}^{2} \zeta_{z}^{2}}\left[\gamma^{(2)}\right]\right\rangle_{\Omega}+\iint_{\Omega} \mu_{\zeta_{x}^{2} \zeta_{y}^{2} \zeta_{z}^{2}}\left[\gamma^{(2)}\right]\left(\mathbf{r}, \mathbf{r}^{\prime}\right) \gamma^{(2)}\left(\mathbf{r r}^{\prime} ; \mathbf{r r}^{\prime}\right) d^{3} r d^{3} r^{\prime}
$$

Differentiating both sides of Eq. (C4) with respect to $\zeta_{x}$, we have

$$
\begin{aligned}
\frac{d \varepsilon_{\zeta_{x}^{2} \zeta_{y}^{2} \zeta_{z}^{2}}\left[\gamma^{(2)}\right]}{d \zeta_{x}}= & \left\langle\frac{d}{d \zeta_{x}} \Psi_{\zeta_{x}^{2} \zeta_{y}^{2} \zeta_{z}^{2}}\left[\gamma^{(2)}\right]\left|\zeta_{x}^{2} \hat{T}_{x}+\zeta_{y}^{2} \hat{T}_{y}+\zeta_{z}^{2} \hat{T}_{z}\right| \Psi_{\zeta_{x}^{2} \zeta_{y}^{2} \zeta_{z}^{2}}\left[\gamma^{(2)}\right]\right\rangle_{\Omega}+\left\langle\Psi_{\zeta_{x}^{2} \zeta_{y}^{2} \zeta_{z}^{2}}\left[\gamma^{(2)}\right]\left|\zeta_{x}^{2} \hat{T}_{x}+\zeta_{y}^{2} \hat{T}_{y}+\zeta_{z}^{2} \hat{T}_{z}\right| \frac{d}{d \zeta_{x}} \Psi_{\zeta_{x}^{2} \zeta_{y}^{2} \zeta_{z}^{2}}\left[\gamma^{(2)}\right]\right\rangle_{\Omega} \\
& +\left\langle\Psi_{\zeta_{x}^{2} \zeta_{y}^{2} \zeta_{z}^{2}}\left[\gamma^{(2)}\right]\left|2 \zeta_{x} \hat{T}_{x}\right| \Psi_{\zeta_{x}^{2} \zeta_{y}^{2} \zeta_{z}^{2}}\left[\gamma^{(2)}\right]\right\rangle_{\Omega}+\iint_{\Omega} \frac{d \mu_{\zeta_{x}^{2} \zeta_{y}^{2} \zeta_{z}^{2}}\left[\gamma^{(2)}\right]\left(\mathbf{r}, \mathbf{r}^{\prime}\right)}{d \zeta_{x}} \gamma^{(2)}\left(\mathbf{r r}^{\prime} ; \mathbf{r r}^{\prime}\right) d^{3} r d^{3} r^{\prime}
\end{aligned}
$$

Comparing Eq. (C3) with Eq. (C5), we found that the first and second terms of Eq. (C1) vanish. Thus, we finally get Eq. (27). 
[1] A. J. Coleman, Rev. Mod. Phys. 35, 668 (1963).

[2] A. J. Coleman, in The Force Concept in Chemistry, edited by B. M. Deb (Van Nostrand Reinhold, New York, 1981), p. 418.

[3] J. Closlowski (Ed.), Many-Electron Densities and Reduced Density Matrices (Kluwer, Dordrecht, 2000).

[4] A. J. Coleman and V. I. Yukalov, Reduced Density Matrices: Coulson's Challenge (Springer-Verlag, Berlin, 2000).

[5] N. I. Gidopoulos and S. Wilson (Eds.), The Fundamentals of Electron Density, Density Matrix, and Density Functional Theory in Atoms, Molecules, and the Solid States (Kluwer, New York, 2003).

[6] R. G. Parr and W. Yang, Density-Functional Theory of Atoms and Molecules (Oxford University Press, New York, 1989), Chap. 2.

[7] P. Ziesche, Phys. Lett. A 195, 213 (1994).

[8] P. Ziesche, Int. J. Quantum Chem. 60, 1361 (1996).

[9] A. Gonis, T. C. Schulthess, J. van Ek, and P. E. A. Turchi, Phys. Rev. Lett. 77, 2981 (1996).

[10] A. Gonis, T. C. Schulthess, P. E. A. Turchi, and J. van Ek, Phys. Rev. B 56, 9335 (1997).

[11] M. Levy and P. Ziesche, J. Chem. Phys. 115, 9110 (2001).

[12] F. Furche, Phys. Rev. A 70, 022514 (2004).

[13] Á. Nagy, Phys. Rev. A 66, 022505 (2002).

[14] Á. Nagy and C. Amovilli, J. Chem. Phys. 121, 6640 (2004).

[15] B. Hetényi, L. Brualla, and S. Fantoni, Phys. Rev. Lett. 93, 170202 (2004).

[16] J. K. Percus, J. Chem. Phys. 122, 234103 (2005).

[17] P. W. Ayers and M. Levy, J. Chem. Sci. 117, 507 (2005).

[18] Á. Nagy, Int. J. Quantum Chem. 106, 1043 (2006).

[19] P. W. Ayers, S. Golden, and M. Levy, J. Chem. Phys. 124, 054101 (2006).

[20] M. Higuchi and K. Higuchi, Physica B 387, 117 (2007).

[21] M. Higuchi and K. Higuchi, Phys. Rev. A 75, 042510 (2007).

[22] M. Higuchi and K. Higuchi, Phys. Rev. B 78, 125101 (2008).

[23] K. Higuchi and M. Higuchi, J. Phys.: Condens. Matter 21, 064206 (2009).

[24] K. Higuchi and M. Higuchi, Phys. Rev. B 82, 155135 (2010).

[25] M. Higuchi and K. Higuchi, Phys. Rev. A 84, 044502 (2011).

[26] E. R. Davidson, Chem. Phys. Lett. 246, 209 (1995).

[27] S. Kh. Samvelyan, Int. J. Quantum Chem. 65, 127 (1997).

[28] M.-E. Pistol, Chem. Phys. Lett. 400, 548 (2004).

[29] P. W. Ayers and M. Levy, Chem. Phys. Lett. 415, 211 (2005).

[30] P. W. Ayers and E. R. Davidson, Int. J. Quantum Chem. 106, 1487 (2006).

[31] P. Gori-Giorgi and A. Savin, Philos. Mag. 86, 2643 (2006).

[32] M.-E. Pistol, Chem. Phys. Lett. 417, 521 (2006).

[33] M.-E. Pistol, Chem. Phys. Lett. 422, 363 (2006).

[34] M.-E. Pistol, Chem. Phys. Lett. 431, 216 (2006).

[35] P. W. Ayers, Phys. Rev. A 74, 042502 (2006).

[36] P. W. Ayers and S. Liu, Phys. Rev. A 75, 022514 (2007).

[37] P. W. Ayers and E. R. Davidson, Adv. Chem. Phys. 134, 443 (2007).

[38] M.-E. Pistol, Chem. Phys. Lett. 449, 208 (2007).

[39] B. Hetényi and A. W. Hauser, Phys. Rev. B 77, 155110 (2008).

[40] N. H. March and R. Santamaria, Int. J. Quantum Chem. 39, 585 (1991).

[41] P. W. Ayers, J. Math. Phys. 46, 062107 (2005).

[42] R. Cuevas-Saavedra and P. W. Ayers, Int. J. Quantum Chem. 109, 1699 (2009).

[43] D. Chakraborty and P. W. Ayers, J. Math. Chem. 49, 1810 (2011).
[44] D. Chakraborty and P. W. Ayers, J. Math. Chem. 49, 1822 (2011). [45] P. Hohenberg and W. Kohn, Phys. Rev. 136, B864 (1964).

[46] W. Kohn and L. J. Sham, Phys. Rev. 140, A1133 (1965).

[47] In general, the computational time depends on the search region of PDs. If the search region is devised appropriately, the computation time for calculating the PD may not always become huge. Actually, by using the recently proposed method, the search region of PDs can be efficiently extended with little increase of the computation time [25].

[48] M. Higuchi and K. Higuchi, Phys. Rev. B 69, 035113 (2004).

[49] K. Higuchi and M. Higuchi, Phys. Rev. B 69, 165118 (2004).

[50] K. Higuchi and M. Higuchi, Phys. Rev. B 71, 035116 (2005).

[51] Á. Nagy, Int. J. Quantum Chem. 106, 1043 (2006).

[52] M. Higuchi and K. Higuchi, Phys. Rev. B 65, 195122 (2002).

[53] O. Gunnarsson and P. Johansson, Int. J. Quantum Chem. 10, 307 (1976).

[54] O. Gunnarsson, M. Jonson, and B. I. Lundqvist, Solid State Commun. 24, 765 (1977).

[55] O. Gunnarsson, M. Jonson, and B. I. Lundqvist, Phys. Rev. B 20, 3136 (1979).

[56] O. Gunnarson and R. O. Jones, Phys. Scr. 21, 394 (1980).

[57] J. A. Alonso and L. A. Girifalco, Solid State Commun. 24, 135 (1977).

[58] J. A. Alonso and L. A. Girifalco, Phys. Rev. B 17, 3735 (1978).

[59] J. P. Perdew and Y. Wang, Phys. Rev. B 33, 8800 (1986).

[60] J. P. Perdew, in Electronic Structure of Solids '91, edited by P. Ziesche and H. Eschrig (Akademie Verlag, Berlin, 1991), p. 11.

[61] J. P. Perdew, K. Burke, and M. Ernzerhof, Phys. Rev. Lett. 77, 3865 (1996).

[62] J. P. Perdew, A. Ruzsinszky, G. I. Csonka, O. A. Vydrov, G. E. Scuseria, L. A. Constantin, X. Zhou, and K. Burke, Phys. Rev. Lett. 100, 136406 (2008); 102, 039902(E) (2009).

[63] V. N. Staroverov, G. E. Scuseria, J. Tao, and J. P. Perdew, Phys. Rev. B 69, 075102 (2004).

[64] S. Liu and R. G. Parr, Phys. Rev. A 53, 2211 (1996).

[65] S. Liu, Á. Nagy, and R. G. Parr, Phys. Rev. A 59, 1131 (1999).

[66] Á. Nagy, S. B. Liu, and R. G. Parr, Phys. Rev. A 59, 3349 (1999).

[67] E. Bene and Á. Nagy, Chem. Phys. Lett. 324, 475 (2000).

[68] S. B. Liu, F. De Proft, Á. Nagy, and R. G. Parr, Adv. Quantum Chem. 36, 77 (2000).

[69] P. W. Ayers, J. B. Lucks, and R. G. Parr, Acta. Phys. Chim. Debricina 34, 223 (2002).

[70] K. Higuchi and M. Higuchi, Phys. Rev. B 74, 195122 (2006); 75, 159902(E) (2007).

[71] M. Higuchi and K. Higuchi, Phys. Rev. B 75, 195114 (2007).

[72] K. Higuchi and M. Higuchi, J. Phys.: Condens. Matter 19, 365216 (2007).

[73] M. Higuchi and K. Higuchi, Phys. Rev. A 81, 042505 (2010).

[74] The explicit form of the KE functional of the DFT is given by

$$
T[\rho]=T_{s}[\rho]+\int_{0}^{1}\left\langle\tilde{\Psi}_{\lambda}|\hat{W}| \tilde{\Psi}_{\lambda}\right\rangle_{\Omega} d \lambda-W[\rho],
$$

where $\left.\tilde{\Psi}_{\lambda}\right\rangle$ is the ground-state wave function that yields the correct ground-state electron density in the system having the scaled Coulomb interaction [6].

[75] A. Görling and M. Levy, Phys. Rev. B 47, 13105 (1993).

[76] A. Görling and M. Levy, Phys. Rev. A 50, 196 (1994).

[77] M. Levy and J. P. Perdew, Phys. Rev. A 32, 2010 (1985).

[78] A. Görling and M. Levy, Phys. Rev. A 45, 1509 (1992). 